\title{
A REVIEW ON ENVIRONMENTS SUPPORTING VIRTUAL ENTERPRISE INTEGRATION
}

\author{
Maria Manuela Cunha \\ Instituto Politécnico do Cávado e do Ave, Portugal, mcunha@ipca.pt \\ Goran D. Putnik \\ Universidade do Minho, Portugal, putnikgd@dps.uminho.pt \\ José Dinis Carvalho \\ Universidade do Minho,Portugal, jdac@dps.uminho.pt \\ Paulo Ávila \\ Instituto Politécnico do Porto, Portugal, pavila@dem.isep.ipp.pt
}

\begin{abstract}
Since the mid nineties, a considerable effort have been undertaken to develop environments to support the Virtual Enterprise life cycle. This effort includes the development of technologies and the development of applications either at the academia or at industrial level to facilitate supply chain management and virtual enterprise integration and its reconfigurability dynamics.

In the paper we intend to present those main contributions, viewing the creation of an environment for the effective and efficient integration of virtual enterprises. We also present the model of the Market of Resources, proposed by the authors, as an electronic brokerage service, designed with this purpose, and discuss its benefits face to other developments.
\end{abstract}

\section{INTRODUCTION}

The goal of the enterprise is to fulfil the customer requirements. Traditionally, the enterprise uses the set of resources existing inside its walls. As this selection domain is relatively limited and of small size, it cannot, in general, provide the desired competitive performances, the enterprise searches for cooperation with other enterprises, corresponding to a shift from "self-centred closed-enterprises" to "global open-enterprises" (Browne \& Zhang, 1999).

Several factors determine the competitiveness of the enterprise, being the most important requirements for competitiveness, the fast adaptability or fast reconfigurability to environmental change (Cunha, Putnik, \& Gunasekaran, 2002). The paradigms satisfying those requisites are the Agile and the Virtual Enterprise ones, which, in the context of the present work, will be designated as the Agile/Virtual Enterprise model (A/V E), corresponding to the Virtual Enterprise model offering the characteristics of the Agile Enterprise. 
The requirements of fast adaptability or reconfigurability implies the ability of (1) flexible and almost instantaneous access to the optimal resources to integrate in the enterprise; (2) design, negotiation, business management and manufacturing management functions independently from the physical barrier of space; and (3) minimisation of the reconfiguration time (Putnik, 2000).

We can identify several phases on the life cycle of an $\mathrm{A} / \mathrm{VE}$ namely: (1) Creation, involving search and selection of partners, and its integration, (2) Operation, (3) Reconfiguration and (4) Dissolution. Reconfiguration happens during $\mathrm{A} / \mathrm{VE}$ operation and implies a redesign of the $\mathrm{A} / \mathrm{VE}$, with search and selection of new partners and integration of the same in a new $A / V E$ instantiation. Fast reconfigurability means that an $\mathrm{A} / \mathrm{VE}$ can have as many instantiations as required in order to keep permanent alignment with the market requirements, and that these reconfigurations happen with a low reconfiguration time and cost (Cunha \& Putnik, 2002). Dissolution is seen as a special case of reconfigurability.

In our work, when referring the A/V E integration, we will implicitly include the upstream phase of search and selecting the partners to integrate. Although much research is being undertaken around the several steps of $A / V E$ integration, its management and co-ordination, insufficient attention has been devoted to the necessity of creating the environment where those processes take place, i.e., the environment to enable an efficient and effective integration, offering strategies for dynamically align the virtual enterprise with business.

This paper reviews some of the most significant contributions towards $\mathrm{A} / \mathrm{V} \mathrm{E}$ integration, namely tools and environments for electronic brokerage, negotiation and integration. Section 2 introduces the main concepts and supporting technologies and techniques. The main contributions towards environments for A/V E integration are addressed in section 3 and section 4 presents the Market of Resources as an environment for A/V E integration. Section 5 concludes the paper, with a simple relationship between techniques/environments and $\mathrm{A} / \mathrm{V} \mathrm{E}$ organisational models.

\section{TECHNOLOGIES AND TECHNIQUES SUPPORTING ENVIRONMENTS FOR VIRTUAL ENTERPRISES INTEGRATION}

Information and Communication Technologies and Internet-based Agent technology are the main technologies for implementation of techniques supporting or contributing to the integration of $\mathrm{A} / \mathrm{VE}^{1}$. These techniques or applications include electronic negotiation, electronic marketplaces and market brokerage. In this section we briefly present the main aspects of Agent technology and of the mentioned techniques. Other fundamental technologies for $\mathrm{A} / \mathrm{VE}$ operation are workflow management systems, collaborative design, virtual product, concurrent engineering.

\subsection{Agent Technologies}

According to a commonly used definition proposed by (Wooldridge, 1997), agents are software systems capable of flexible, autonomous action in some environment in order to meet its design objectives. Agents consists on software than can travel over 
networks, activate and control remote programs, interrogating host Web sites, interacting with other agents and return to source with information.

Some specific domains where, according to (Oliveira, 1999), the agent-based solution proved to be appropriate include ${ }^{2}$ :

- Softbots, (software robots) are agents living in virtual environments, having access to multiple heterogeneous and geographically distributed information sources as Internet, to perform active searches for relevant information, like Web pages containing information considered important for its user.

- Virtual Organizations, in Internet-based marketplaces and in the integration virtual enterprises, agents can search for partners to integrate a supply chain or a virtual organisation, negotiate, organise auctions and manage bids.

- Electronic Commerce, where agents can search for products /services, negotiate and manage the transaction, and organise bids, in an electronic marketplace.

Other applications could be referred, but are out of our scope of interest.

\subsection{Electronic Automated Negotiation (e-Negotiation)}

(Beam \& Segev, 1997) define electronic automated negotiation as the process by which two or more parties multilaterally bargain resources for mutual intended gain, using the tools and techniques of electronic commerce in networked computers. Automated negotiation is difficult, namely because of: (1) the need for an ontology for categorizing objects, such that they are semantically meaningful to a software agent, and (2) the need for a algorithmic negotiation strategy.

The most basic form of e-Negotiation is no negotiation at al, i.e., fixed-price $\underline{\text { sale, }}$ where the seller offers the goods or services through a catalogue at take-it-orleave-it prices. Other styles of negotiation are auctions, bilateral bargaining (which involves making proposals and counter-proposals until agreement is reached), combinatory auctions or direct negotiation within a small set of eligible resource providers (bilateral bargaining within a smaller domain manually performed).

Agent technology plays an important role in the automation of e-Negotiation.

\subsection{Electronic Marketplaces (e-Marketplaces)}

Firms exist to reduce the costs of negotiating, monitoring and executing transactions that are necessary to produce or acquire goods and services on the market. Market is the mechanism that allows buyers and sellers to change things. Its main characteristic is to link buyers and sellers to define prices and quantities. An Electronic Marketplace is an Internet-based environment where participants (buyers and sellers) can meet to exchange goods and services or to cooperate in order to achieve a common business goal. The economic benefits to firms are participation and value creation in wider market-like relationships organised electronically while reducing costs through automation of a higher volume of transactions.

Compared with other electronic procurement solutions, e-Marketplaces represent a relatively neutral position between buyer and seller, providing services to both sides of a transaction (Segev, Gebauer, \& Beam, 1998). In a multiagent supported e-Marketplaces, where agents can meet to exchange services or trade with a variety of merchandise, the agents can represent various organisations and companies. A 
negotiation on price and quantity and a contract between two agents can be set up and the transaction carried out within minutes or even seconds.

\subsection{Market Brokerage}

The concept of broker, capable of acting on behalf of a customer in guiding the selection of the most suitable product, has long been well known and the extension of this concept to the electronic marketplace is a natural progression.

Market brokerage is a core concept to overcome the current limitations of e-Marketplaces, namely the problems of semantic matching and the coordination of the selection of resources to integrate in an A/V E (Cunha, Putnik, \& Ávila, 2000). Appropriate brokerage services should support new market-led relationships between producers and consumers, where the large number of suppliers and customers are geographically separated, where there are many comparable products or when prices and product features or models change rapidly. A purpose of electronic brokerage is the reduction of costs of search and discovery mediated by a third party (the broker) for buyers and sellers of physical or logical goods and services within an e-marketplace (Bichler, 1998). Market brokerage can be performed with different degrees of automation, from manual or fully automated using intelligent agent technology. The importance of brokerage is discussed in the Reference Model proposed by (Putnik, 2000), and a detailed broker functions taxonomy can be found in (Ávila, Putnik, \& Cunha, 2002).

\section{ENVIRONMENTS TOWARDS VIRTUAL ENTERPRISE}

We refer in this section some relevant environments contributing for $A / V E$ integration, supporting any or all of the following functions: A/VE design, selection, negotiation and integration of resources into $\mathrm{A} / \mathrm{V} E$ enterprises, and coordination functions. Examples include electronic marketplaces and agent-based brokerage.

\subsection{Electronic Marketplaces}

Recent years have seen a dramatic emerging of e-marketplaces for business opportunities and Internet-based online auctions. There exist several Electronic markets operating in a variety of industries, from industrial metals, chemicals, construction, textile and many others, distinguished by factors such as main focus or scope, revenue strategies, restriction to entry or openness (limitation on number of participants), contents in databases or references (degree of distributiveness of catalogues), degree of automation in negotiation, support to transactions, etc.

A valuable example is Covisint (http://www.covisint.com), an e-marketplace consisting on a Virtual Supplier Network (a service encompassing the complete interaction between suppliers or suppliers and their customers, using network technology) specifically created for the automotive industry. The service is projected to be a one-stop-shop, supporting procurement transactions, pre-production collaborative engineering and exchange of information during production or for supply chain management. Covisint was officially announced in December 2000 as an independent company, created by Ford, Chrysler, General Motors, Renault and Nissan and a number of development partners. 


\subsection{Agent-based Brokerage and Marketplaces}

Agent-based approaches have been already applied for enterprise integration, manufacturing production planning, scheduling and process control, material handling for one decade. Several researchers have proposed the organisation of the supply chain as a network of cooperating, intelligent agents, just to mention:

- (Sauter \& Parunak, 1999) proposed the Agent Network for Task Scheduling (ANTS) architecture with techniques inspired by both human institutions and insect colonies, in which large populations of simple agents support scheduling in supply chains;

- The project MetaMorph II (Shen \& Norrie, 1998) proposed the use of hybrid agent-based architecture for manufacturing enterprise integration and supply chain management, i.e., a mediator-centric architecture to integrate partners, suppliers and customers dynamically with the main enterprise through their respective mediators within a supply chain network via the Internet.

- (Shen, Ulieru, Norrie, \& Kremer, 1999) presented a case study of supply chain management for a large manufacturing enterprise, CASA, based on Collaborative Agent System Architecture;

The implementation of marketplaces based on Agents is also usual. Several projects have been found, such as:

- The experiment at BT Laboratories intends to build a broker-less marketplace based on agents (Collis \& Lee, 1998a, 1998b). In this scenario, each agent will have the ability to contact every other agent and negotiate to buy and sell directly; this means there is no role for intermediary agents such as brokers.

- The OFFER Project (Object Framework for Electronic Requisitioning) (Bichler, Beam, \& Segev, 1998) is an object framework for business-to-business electronic commerce that contains a CORBA-based electronic broker who assists the user in two ways during a market transaction: first, it helps search in many, often unknown electronic catalogs of suppliers; second, it provides auction mechanisms to support price negotiation between buyers and sellers.

- The Global Electronic Marketplace (GEM) (Rachlevsky-Reich \& Ben-Shaul, 1999 ) is an electronic commerce platform containing a complex and distributed structure of trading facilities based on brokers and a central market server. GEM simulates a trading floor by bringing together and bids collected by the brokers.

- MAGMA (Minnesota Agent Marketplace Architecture) is an architecture for Agent-based virtual marketplace, providing all services essential to agent-based commercial activities, available through an open-standard messaging API, which allows use of a heterogeneous set of agents independently of platform and language (Tsvetovatyy, Gini, Mobaster, \& Wieckowski, 1997).

- In the model for negotiation in virtual organisations formation proposed by (Oliveira \& Rocha, 2000), when a specific consumer's need is identified, it is created a new agent that will formulate an announcement for goal satisfaction in the electronic marketplace, will receive and evaluate bids from potential suppliers of the product and negotiate in order to integrate the partnership.

However it was not possible to find many applications of this technology covering the whole process of enterprise selection, negotiation, integration, and coordination of integration. 


\section{MARKET OF RESOURCES AS A VIRTUAL ENTERPRISE INTEGRATION ENVIRONMENT}

Three relevant requisites are identified in relation with the process of A/V E design or integration: (1) Flexible and almost instantaneous access to the independent candidate resources to integrate a virtual enterprise, negotiation process between them, selection of the optimal combination and its integration; (2) Design, negotiation, business management and manufacturing management functions independently from the physical barrier of space; and (3) Minimisation of the reconfiguration and integration time and cost. These requisites imply the existence of a market of independent candidate resources for integrating a virtual enterprise, able: (1) to provide the environment and technology for efficient access to resources, efficient negotiation between them and its efficient integration; and (2) to provide a domain for selection of participant resources in a virtual enterprise, large enough to assure a good combination of resources.

The concept of Market of Resources was introduced by the authors in (Cunha et al., 2000), as an electronic and virtual market, mediating offer and demand of resources to dynamically integrate in an A/V E. Enterprises (resources providers) subscribing the Market of Resources, make their resources available as potential servers /partners for A/V E integration. The Market of Resources supports the A/V E model proposed in the Virtual Enterprise Reference Model (BM-VEARM) by (Putnik, 2000).

The service provided by the Market of Resources is supported by (1) a knowledge base of resources and results of the integration of resources in previous $\mathrm{A} / \mathrm{V} \mathrm{E},(2)$ a normalised representation of information and (3) intelligent agent and algorithms, (4) a brokerage service and (5) regulation, i.e. management of negotiation and integration processes. It is able to offer (1) knowledge for A/V E selection of resources, negotiation and its integration, (2) specific functions of A/V E operation management, and (3) contracts and formalising procedures to assure the accomplishment of commitments, responsibility, trust and deontological aspects, envisaging that the integrated $\mathrm{A} / \mathrm{VE}$ accomplishes its objectives of answering to a market opportunity. The environment supports not only the integration process, but, what is most important when the fast and proficient reaction to change is a key element, is able to effectively support dynamic integration, which is the main reason for the concept of Market of Resources as an institution.

\section{CONCLUSIONS}

Although it was not possible an exhaustive review, we tried to present the main developments and contributions towards the creation of environments able to support the processes of A/V E (design and) integration. We were able to conclude that all the required technologies and techniques already exist, most of them dispersedly developed, as well as many valuable applications are already in operation, however there is missing what we designate as an adequate environment to support the inherent need of dynamics required by the emerging paradigm of 
A/V E. Market of Resources was conceived precisely to "boost" the dynamics as well as to support the high dynamics of the A/V E reconfiguration and integration ${ }^{3}$.

The table bellow summarises the contribution of each technology/technique and of each environment on the main Virtual Enterprise models: Electronic Commerce, Supply Chain, Extended Enterprise, BM_VEARM and OPIM (One Product Integrated Manufacturing), (Putnik, Guimarães, \& Silva, 1996). It is also referred the importance of reconfigurability dynamics for each VE model.

The Market of Resources is proposed as an ideal environment to support the requirement of fast adaptability of the most dynamic VE models life cycle (BM_VEARM and OPIM), while the other environments are designed to cope only VE models that do not require a high reconfigurability dynamics.

\begin{tabular}{|c|c|c|c|c|c|c|c|c|c|}
\hline & \multicolumn{4}{|c|}{ Technologies / Techniques } & \multicolumn{3}{|c|}{ Environments } & \multirow{2}{*}{$\begin{array}{c}\text { Importance } \\
\text { of reconfig } \\
\text { dynamics }\end{array}$} \\
\hline & & \begin{tabular}{|l|} 
Agents \\
Technol
\end{tabular} & \begin{tabular}{|l|} 
Electr. \\
Negot.
\end{tabular} & \begin{tabular}{|l|} 
Market \\
places
\end{tabular} & $\begin{array}{l}\text { Market } \\
\text { Broker }\end{array}$ & \begin{tabular}{|l|} 
Electron. \\
Marketp.
\end{tabular} & \begin{tabular}{|c|} 
Agent \\
Broker.
\end{tabular} & $\begin{array}{l}\text { Market } \\
\text { Resour. }\end{array}$ & \\
\hline \multirow{5}{*}{ 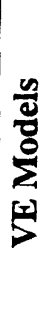 } & e-commerce & & $\mathrm{x}$ & $\mathrm{x}$ & & $\mathbf{x}$ & $\mathrm{x}$ & & low \\
\hline & Supply chain & $\mathbf{x}$ & $\mathbf{x}$ & $\mathbf{x}$ & & $\mathbf{x}$ & $\mathrm{x}$ & & medium \\
\hline & \begin{tabular}{|l|} 
Extended \\
Enterprise \\
\end{tabular} & $\mathbf{x}$ & $\mathrm{x}$ & $\mathbf{x}$ & $\mathbf{x}$ & $\mathrm{x}$ & $\mathrm{x}$ & & medium \\
\hline & BM_VEARM & $\mathrm{x}$ & $\mathrm{x}$ & $\mathrm{x}$ & $\mathrm{x}$ & & & $\mathbf{x}$ & high \\
\hline & OPIM & $\mathbf{x}$ & $\mathrm{x}$ & $\mathbf{x}$ & $\mathbf{x}$ & & & $\mathbf{x}$ & high \\
\hline \multicolumn{6}{|c|}{ Ability to support fast reconfigurability } & low & low/med & high & \\
\hline
\end{tabular}

\section{REFERENCES}

1. Ávila, P., Putnik, G. D., \& Cunha, M. M. (2002). Brokerage Function in Agile/Virtual Enterprise Integration - A Literature Review. In L. M. Camarinha-Matos (Ed.), Collaborative Business Ecosystems and Virtual Enterprises: Kluwer Academic Publishers.

2. Beam, C., \& Segev, A. (1997). Automated Negotiations: A Survey of the State of the Art. Retrieved, June 2001, from the World Wide Web: http://www.haas.berkeley.edu/ citm/nego-proj.htmV

3. Bichler, M. (1998). An Electronic Broker to Business-to-Business Electronic Commerce on the Internet. International Journal of Cooperative Information Systems, 7(4).

4. Bichler, M., Beam, C., \& Segev, A. (1998). OFFER: A Broker-Centered Object Framework for Electronic Requisitioning, Proceedings of IFIP Conference: Trends in Electronic Commerce. Hamburg, Germany.

5. Browne, J., \& Zhang, J. (1999). Extended and Virtual Enterprises: similarities and differences. International Journal of Agile Management Systems, 1/I, 30-36.

6. Collis, J. C., \& Lee, L. C. (1998a, May 1998). Building Electronic Marketplaces with the Zeus Agent Toolkit. Paper presented at the Workshop of the Agents'98 2nd International Conference on Autonomous Agents, Minneapolis, USA.

7. Collis, J. C., \& Lee, L. C. (1998b). Building Electronic Marketplaces with the Zeus Agent Toolkit. Suffolk, UK: Intelligent Systems Research Unit, BT Laboratories.

8. Cunha, M. M., \& Putnik, G. D. (2002). Discussion on Requirements for Agile/Virtual Enterprises Reconfigurability Dynamics: The Example of the Automotive Industry. In L. M. Camarinha-Matos (Ed.), Collaborative Business Ecosystems and Virtual Enterprises: Kluwer Academic Publishers.

9. Cunha, M. M., Putnik, G. D., \& Ávila, P. (2000). Towards Focused Markets of Resources for Agile / Virtual Enterprise Integration. In H. Afsarmanesh (Ed.), Proceedings of the 4th IEEE/IFIP International Conference on Information Technology for Balanced Automation Systems in Manufacturing and Transportation. Berlin: Kluwer Academic Publishers. 
10. Cunha, M. M., Putnik, G. D., \& Gunasekaran, A. (2002). Market of Resources as an Environment for Agile / Virtual Enterprise Dynamic Integration and Business Alignment. In A. Gunasekaran (Ed.), Knowledge and Information Technology Management in the 21st Century Organisations: Human and Social Perspectives. London: Idea Group Publishing.

11. Jennings, N. R., Farantin, P., Johnson, M. J., O'Brien, P., \& Wiegand, M. E. (1996). Agent-based Business Process Management. International Journal of Cooperative Information Systems, 5(2-3), 105-130.

12. Jennings, N. R., \& Wooldridge, M. (1998). Applications of Intelligent Agents. In M. Wooldrodge (Ed.), Agent Technology: Foundations, Applications and Markets: Springer-Verlag.

13. MIT_Media_Lab. (2001, May 2001). Projects. Software Agents Group, Massachusetts Institute of Technology. Retrieved December 2001, from the World Wide Web:

14. Oliveira, E. (1999). Applications of Intelligent Agent-Based Systems. Paper presented at the $4^{\circ}$ Simpósio Brasileiro de Automação Inteligente, SBAI, São Paulo, SP.

15. Oliveira, E., \& Rocha, A. P. (2000). Agents advanced features for negotiation in Electronic Commerce and Virtual Organisations formation process. In C. Sierra (Ed.), Agent Mediated Electronic Commerce, the European AgentLink Perspective, Lectures Notes in Artificial Intelligence (Vol. 1991, pp. 77-96): Springer-Verlag.

16. Putnik, G. (2000). BM_Virtual Enterprise Architecture Reference Model. In A. Gunasekaran (Ed.), Agile Manufacturing: 21st Century Manufacturing Strategy (pp. 73-93). UK: Elsevier Science Publ.

17. Putnik, G. D., Guimarães, P. F., \& Silva, S. d. C. (1996). Virtual Enterprise / OPIM Concepts: an institutionalization framework. In $\mathrm{H}$. Afsarmanesk (Ed.), Proceedings of the 2nd IEEE/ECLA/IFIP International Conference on Architectures and Design Methods for Balanced Automation Systems (pp. 391-400). Portugal: Chapman \& Hall.

18. Rachlevsky-Reich, B., \& Ben-Shaul, I. (1999). GEM: A Global Electronic Market System. Information Systems, 24(6), 495-518.

19. Sauter, J. A., \& Parunak, R. (1999, May 1999). ANTS in the Supply Chain. Paper presented at the Agent's 99 Workshop on Agent-Based Decision Support for Managing the Internet-Enabled Supply Chain, Seattle.

20. Segev, A., Gebauer, J., \& Beam, C. (1998). Procurement in the Internet Age - Current Practices and Emerging Trends (Results from a field study) (CMIT Working Paper 98-WP-1033). Berkeley, CA: Fisher Center for Management and Information Technology, University of California at Berkeley.

21. Shen, W., \& Norrie, D. H. (1998). An Agent-Based Approach for Manufacturing Enterprise Integration and Supply Chain Management. In G. J. e. al (Ed.), Globalization of Manufacturing in Digital Communications Era of the 21st Century: Innovation, Agility and the Virtual Enterprise (pp. 579-590): Kluwer Academic Publishers.

22. Shen, W., Ulieru, M., Norrie, D. H., \& Kremer, R. (1999, May 1999). Implementing the InternetEnabled Supply Chain Through a Collaborative Agent System. Paper presented at the Agent's 99 Workshop on Agent-Based Decision Support for Managing the Internet-Enabled Supply Chain, Seattle.

23. Tsvetovatyy, M., Gini, M., Mobaster, B., \& Wieckowski, Z. (1997). MAGMA: An Agent-based Virtual Market for Electronic Commerce. Journal of Applied Artificial Intelligence, 11(Special Issue on Intelligent Agents).

24. Wooldridge, M. (1997). Agent-based Software Engineering. IEE Proc. Software Engineering, 144, 26-37.

25. Wooldridge, M., \& Jennings, N. R. (1995). Agent Theories, Architectures and Languages: A Survey. In N. R. Jennings (Ed.), Intelligent Agents: Theories, Architectures and Languages (Vol. LNAI Vol 890 , pp. 1-39). Heidelberg, Germany: Springer-Verlag.

${ }^{1}$ Understanding A/VE integration as including the processes of selection and negotiation between candidate resources and the integration of the selected resources.

${ }^{2}$ For detailed descriptions of agents theory, architectures and languages we suggest (Wooldridge \& Jennings, 1995) and for agents applications, (Jennings, Farantin, Johnson, O'Brien, \& Wiegand, 1996; Jennings \& Wooldridge, 1998; Oliveira, 1999), among many other valuable sources of information. The MIT Media Lab site provides interesting and valuable information on underway research projects at MIT's Software Agents Research Group (MIT_Media_Lab, 2001).

${ }^{3}$ We did not address the issues of emerging standards for interoperability and information interchange, Internet protocols and other essential infrastructures, as we were concerned, in the present work, with the technologies and environments supporting A/V E integration. 